\title{
SOBREVIVENCIA DE LAS PYME EN CHILE*
}

\section{Roberto Álvarez y Sebastián Vergara}

\begin{abstract}
Este estudio analiza la relación entre probabilidad de sobrevivencia y tamaño de plantas en las pequeñas y medianas empresas (PYME). Utilizando una base de datos para la industria manufacturera que cubre el período 1979-1999, se analiza si las PYME han experimentado efectos negativos en su probabilidad de sobrevivencia en el tiempo. Los resultados no revelan evidencia en este sentido. Para profundizar el análisis, se estudian diferencias en la probabilidad de sobrevivencia distinguiendo el grado de exposición al comercio internacional de los sectores manufactureros. Por otro lado, también se analiza la relación entre sobrevivencia y tamaño en 25 sectores industriales. Los resultados sugieren (i) que la mayor competencia externa aumenta la probabilidad de sobrevivencia de las PYME y (ii) que hay gran heterogeneidad en los distintos sectores industriales en lo que concierne al efecto del tamaño en la sobrevivencia. Esta evidencia sugiere algunas dudas respecto a la implementación de políticas de apoyo a las PYME que no consideran las diferentes realidades sectoriales ni las diferencias entre plantas pequeñas y medianas.
\end{abstract}

RoBerto Álvarez. Economista de la Universidad de Chile y Doctor en Administración de la Universidad de California, Los Angeles (UCLA). Economista Senior de la Gerencia de Investigación Económica, Banco Central de Chile.

Sebastián Vergara. Economista de la Universidad de Chile, actualmente cursa el Master of Arts en Economía Internacional de la Universidad de Sussex, Reino Unido. Economista de la División de Desarrollo Productivo y Empresarial de CEPAL.

* Agradecemos los comentarios recibidos en seminarios en el Departamento de Administración de la Universidad de Chile, en la Universidad Adolfo Ibáñez y de un árbitro anónimo de Estudios Públicos. Las opiniones, errores y omisiones son de nuestra exclusiva responsabilidad y no necesariamente reflejan la posición del Banco Central de Chile ni la de sus Consejeros.

Estudios Públicos, 107 (invierno 2007). 


\section{Introducción}

$\mathrm{D}$

urante los últimos años ha existido un interesante debate en Chile respecto a la necesidad de apoyar el desarrollo de las PYME. En la última campaña presidencial y recientemente en la discusión de qué hacer con los recursos originados por el alto precio del cobre, no son pocos los analistas que han propuesto incrementar los recursos para el fomento de este segmento de empresas. Sin embargo, la mayoría de estas propuestas se basan en una serie de creencias sobre el desempeño de las PYME y los problemas que éstas enfrentan. Salvo algunos trabajos, como el de Benavente et al. (2005) y Cabrera et al. (2002), existen pocos estudios empíricos que validen o rechacen estas creencias ${ }^{1}$.

Este tipo de debates no sólo se ha generado en Chile, sino también en otros países en desarrollo. El proceso de globalización de las últimas décadas ha suscitado una interesante discusión en torno a sus efectos en el desempeño de las empresas más pequeñas. Por una parte, se argumenta que las empresas de menor tamaño relativo son afectadas negativamente por la mayor globalización debido a su posición desventajosa para enfrentar la creciente competencia en los mercados locales, y más aún en países en desarrollo que presentan variados problemas en el funcionamiento y profundidad de sus mercados de capitales. La UNCTAD (2004) por ejemplo plantea que: "La liberalización comercial incrementa la capacidad de las empresas transnacionales para penetrar mercados externos y poco desarrollados, y hace aún más difícil que las PYME sobrevivan o mantengan su posición en el mercado local y, si corresponde, en el mercado global”.

Esta visión concuerda con la creencia tradicional de que la liberalización y la mayor competencia son especialmente hostiles para las PYME. Esta idea se asocia a varias desventajas de "ser pequeño". Entre ellas, los altos costos de aprendizaje en los mercados externos y la adopción de nuevas tecnologías, y las escasas posibilidades de generar economías de escala. Asimismo, existen argumentos respecto a las asimetrías de información en los mercados de capitales — riesgo moral y selección adversa- que afectan las posibilidades de las empresas más pequeñas para acceder a financiamiento (Cooley y Quadrini, 2001). En estas circunstancias se argu-

${ }^{1}$ Otras recientes excepciones son Álvarez y Vergara (2006), que estudian conjuntamente los determinantes de la sobrevivencia y el crecimiento del empleo por segmento de empresas, y Álvarez y Crespi (2001), quienes estudian qué determina la importancia de las PYME en los sectores manufactureros. Benavente y Ferrada (2003) realizan un análisis similar a este trabajo, pero no estudian explícitamente cómo la sobrevivencia de las PYME ha cambiado en el tiempo ni explotan diferencias sectoriales. 
menta que "para competir globalmente es necesario ser grande" (Chandler, 1990).

En contraposición a esta visión tradicional, algunos autores muestran las ventajas de las PYME para adaptarse a la creciente competencia externa. Audretsch (2002), por ejemplo, enfatiza el rol de las empresas pequeñas como fuente de innovación, crecimiento y competitividad. Las PYME tendrían ventajas relativas asociadas a su mayor flexibilidad y menor burocracia en comparación con las empresas más grandes, lo que incentivaría una mayor innovación en procesos y productos. Similarmente, Acs et al. (1996) argumentan que las PYME serían más aptas para promover la innovación. Ello debido a que en empresas más pequeñas es más probable que los propios innovadores puedan capturar los beneficios de sus innovaciones. En las empresas grandes, por el contrario, los innovadores tienen derechos de propiedad limitados y los nuevos productos y procesos suelen pertenecer a la empresa. En cambio, Cohen y Kepler (1996) concluyen que mientras las PYME pueden ser superiores en lo que respecta a generación de nuevo conocimiento, las empresas más grandes estarían en mejor posición para apropiarse de los beneficios derivados de estas innovaciones. Audretsch (1995), sin embargo, entrega evidencia de que mientras las empresas más grandes exhiben mayor gasto en I\&D, son las PYME las que participan en mayor medida en la innovación de productos.

Es importante analizar la evidencia empírica en que se sustentan estas distintas visiones del rol y funcionamiento de las PYME. Dependiendo del sustento empírico de cada una de ellas, serán más o menos justificadas políticas de apoyo específicas (por ejemplo, financiamiento, asociatividad, creación de redes, reducción y/o facilitación de trámites, ley de quiebras, etc.). De este modo, en la medida que exista más y mejor información respecto del funcionamiento de las PYME, se podrán desarrollar e implementar programas de apoyo más eficientes.

De hecho, en los últimos años las políticas de apoyo a las PYME han recobrado importancia no sólo en Chile sino en toda América Latina. Mientras a comienzos de la década de los noventa predominaba en la región la visión de que la mejor política para las PYME era justamente "no hacer política”, desde la segunda mitad de la década se aprecia una renovada visión —política e institucional— para el desarrollo de nuevos esquemas de apoyo y fomento ${ }^{2}$.

${ }^{2}$ La política de apoyo a las PYME en Chile ha tomado renovado impulso con la implementación en 1991 del Programa de Apoyo a la Pequeña y Mediana Empresa, por parte de la Corfo. 
A pesar de las visiones conceptuales contrapuestas y del desarrollo de políticas de fomento a las PYME hay escasa evidencia empírica de su validez en países en desarrollo. Algunas excepciones son, por ejemplo, Peres y Stumpo (2000), quienes comparan el desempeño de las PYME en términos de producción, empleo y productividad para un grupo de países en América Latina antes y después de las reformas estructurales. Estos autores concluyen que, a pesar de la alta heterogeneidad en el desempeño de las PYME entre países, estas firmas no han sido los agentes "perdedores" en el nuevo modelo económico. Sin embargo, su análisis es agregado y poco se puede decir del comportamiento al nivel de las firmas o para un país en particular.

Este estudio contribuye a la discusión mostrando evidencia empírica respecto de cómo la sobrevivencia de las PYME ha ido cambiando a través del tiempo. Dado que la economía chilena ha emprendido un profundo proceso de liberalización y reformas estructurales, es interesante analizar si el riesgo de muerte para las PYME se ha ido incrementando.

Adicionalmente, se estudia la probabilidad de sobrevivencia de estas empresas en sectores que difieren en su grado de exposición a la competencia externa. Si, como ha argumentado la UNCTAD (2004) y Chandler (1990), la liberalización comercial afecta de manera desproporcionada a las pequeñas empresas, entonces se deberían observar cambios en la relación entre tamaño de firmas y sobrevivencia, dependiendo de qué tan importante es el grado de intercambio comercial con el resto del mundo.

Para investigar estos efectos aplicamos la metodología típicamente usada en economía laboral, distinguiendo lo que puede denominarse como un grupo de tratamiento y otro de control (Hanson, 2005). Siguiendo esta literatura, definimos como grupo de tratamiento a aquellas firmas en sectores industriales que más han incrementado sus flujos comerciales (sectores de alta exposición a la competencia externa), y un grupo de control conformado por plantas en sectores en los cuáles el intercambio con el resto del mundo se ha incrementado relativamente poco (sectores de baja exposición). Además, investigamos la relación entre sobrevivencia y tamaño en 25 sectores industriales. Ello permite establecer en qué medida la relación negativa entre tamaño y sobrevivencia difiere por industrias.

Este trabajo está organizado en cinco secciones. La segunda sección describe la información estadística disponible y motiva el ejercicio empírico mostrando tasas de sobrevivencia según tamaño de plantas y períodos. La tercera sección presenta la metodología y discute las principales hipótesis. La cuarta sección discute los resultados econométricos. En concordancia con estudios previos para otras economías, la evidencia revela que las PYME tienen mayores probabilidades de salida que las empresas de mayor 
tamaño. No obstante, no es posible afirmar que la probabilidad de sobrevivencia haya cambiado significativamente en el tiempo. Más interesantes aún son los resultados entre sectores: se encuentra que las plantas industriales en sectores "expuestos a la competencia externa" tienen mayores probabilidades de sobrevivencia, y que las PYME no son negativamente afectadas por la mayor competencia. De hecho, parece que el efecto negativo de ser pequeño es menor en industrias más expuestas al comercio internacional. La quinta sección presenta las principales conclusiones.

\section{Datos y características generales}

El análisis empírico se basa en la información estadística proveniente de la Encuesta Nacional Industrial Anual (ENIA), realizada por el Instituto Nacional de Estadísticas (INE), que cubre las plantas manufactureras con 10 o más empleados. Utilizamos información para el período 1979-1999.

El INE actualiza año a año la encuesta incorporando plantas que iniciaron operaciones durante el año y excluyendo aquellas plantas que dejaron de operar. Para cada planta, la ENIA recopila información respecto de producción, valor agregado, ventas, empleo, salarios, exportaciones, inversión, depreciación, uso de energía y otras características. Asimismo, las plantas son clasificadas de acuerdo a la Clasificación Internacional Industrial Uniforme (CIIU), Revisión 2. Usando deflactores para cada uno de los sectores a 4 dígitos de la CIIU, las variables monetarias fueron convertidas en pesos constantes de 1985.

Definimos la salida de una firma utilizando información de la presencia de la planta en un año $t$ y su ausencia en algún año $t+s$. Sin embargo, como lo muestran Benavente y Ferrada (2003), la entrada y salida de firmas puede deberse a otras razones que no constituyan necesariamente el nacimiento ni el quiebre de empresas ${ }^{3}$. Además, como el criterio de selección es plantas con más de 10 trabajadores, aquellas plantas que reducen su tamaño por debajo de este límite se codificarían erróneamente como salida de firmas.

Este tipo de problemas con la base de datos implica que los resultados deban interpretarse con cuidado. Primero, dado que la información excluye un segmento importante de microempresas, poco tenemos que decir

${ }^{3}$ Entradas y salidas falsas pueden estar asociadas con plantas que alcanzan niveles de empleo inferiores a 10 trabajadores. Además, ciertas salidas de firmas puede ser resultado de plantas que: a) no estaban localizadas al momento de la encuesta, b) no tuvieron movimientos de capital, c) paralizaron operaciones, d) estaban bajo investigación por parte del Servicio de Impuestos Interno (SII) o e) se fusionaron con otra planta. 
respecto a cómo la probabilidad de sobrevivencia para estas empresas ha cambiado a través del tiempo y entre industrias. Segundo, nuestra medida de probabilidad de sobrevivir puede tener un sesgo a la baja al considerar como salida la caída por debajo del límite de 10 trabajadores, la no localización de plantas al momento de la encuesta o la fusión con otra planta. Aun cuando estos errores de medición son potencialmente importantes, en términos econométricos su impacto tendería a ser menor considerando que éstos están concentrados en nuestra variable dependiente. Por otro lado, la utilización de un panel de datos minimiza en cierto sentido estos errores de medición. De hecho, sólo tendríamos un problema considerable en la medida en que el sesgo cambie sistemáticamente en el tiempo o difiera sistemáticamente entre las diferentes industrias ${ }^{4}$. Finalmente, aun cuando estos problemas sean potencialmente importantes, los resultados parecen sugerir que los problemas de medición no afectan significativamente la calidad de nuestros hallazgos. En general, éstos son bastante concordantes con la literatura previa.

Otra precisión necesaria es lo que se entiende por pequeña empresa y mediana empresa. Existen principalmente dos criterios para definirlas: las ventas (en UF) o el empleo total (número de trabajadores). En este trabajo seguimos el segundo criterio. Se clasifica una planta como pequeña cuando tiene un máximo de 50 trabajadores, mientras que las plantas medianas son aquellas que tienen entre 50 y 150 trabajadores. Nuestro grupo de comparación será el segmento de plantas grandes, las que tienen más de 150 trabajadores.

Una tercera precisión se refiere a la salida de firmas. Dado que queremos estudiar cómo la salida de firmas ha ido cambiando a través del tiempo, escogimos una periodicidad de 5 años de sobrevivencia, que es concordante con nuestra intención de estudiar cambios más de mediano plazo y no fluctuaciones año a año. Para chequear la robustez de los resultados, también mostramos los resultados de sobrevivencia en períodos de 3 años ${ }^{5}$.

${ }^{4}$ Hemos tratado de minimizar el impacto de estos problemas de varias maneras. Primero, excluimos a todas aquellas firmas que presentan una salida de la encuesta pero vuelven a aparecer en un año posterior. Segundo, hicimos las estimaciones excluyendo a aquellas firmas que en algún año tengan menos de 15 trabajadores (haciendo más alto el límite que impone el INE). Tercero, usamos la base de Benavente y Ferrada (2003), que ha "limpiado" la base de datos de entradas y salidas falsas. Nuestros resultados son robustos a estas modificaciones.

${ }^{5}$ En general, los resultados no dependen de la periodicidad escogida. Usando también datos de plantas manufactureras chilenas para estudiar la respuesta de multinacionales a la crisis económica 1998-1999, Álvarez y Görg (2007) muestran que sus resultados en términos de sobrevivencia y empleo son robustos al usar datos anuales. 
En el Cuadro $\mathrm{N}^{\circ} 1$ se presenta la información de la probabilidad de sobrevivencia según diferentes tamaños de firmas y diferentes períodos de tiempo $^{6}$. Es posible destacar tres principales hechos. Primero, conforme a lo esperable según la evidencia internacional, la sobrevivencia de las plantas tiende a disminuir con el tamaño. En promedio, la probabilidad de sobrevivencia es $66 \%$ para las plantas pequeñas, $76 \%$ para las medianas y $82 \%$ para las grandes. Segundo, la tasa de sobrevivencia es menor en los períodos correspondientes al inicio y al final de la muestra ${ }^{7}$. En cambio, en los períodos intermedios - caracterizados por la liberalización comercial y alto crecimiento económico- la tasa de sobrevivencia tiende a ser mayor. Tercero, se observa un comportamiento heterogéneo por segmento de plantas. Durante los períodos de rápido crecimiento, 1984-1989 y 1989-1994, la probabilidad de sobrevivencia se incrementó para las pequeñas empresas, pero se redujo para las empresas medianas y grandes.

En resumen, conforme con la evidencia internacional, se tiene que las PYME exhiben, en general, una menor probabilidad de sobrevivencia ${ }^{8}$. Sin embargo, esta evidencia preliminar no parece sugerir que haya existido una reducción en sus oportunidades de sobrevivir a través del tiempo. En el último período de la muestra existe una reducción en la tasa de sobreviven-

CUADRO No $1: \quad$ PROBABILIDAD DE SOBREVIVENCIA SEGÚN TAMAÑO Y PERÍODOS

\begin{tabular}{lcccc}
\hline Período & Total & Pequeñas & Medianas & Grandes \\
\hline & & & & \\
$1979-1984$ & 0,63 & 0,60 & 0,73 & 0,83 \\
$1984-1989$ & 0,77 & 0,74 & 0,84 & 0,93 \\
$1989-1994$ & 0,79 & 0,77 & 0,81 & 0,86 \\
$1994-1999$ & 0,58 & 0,54 & 0,64 & 0,66 \\
Promedio simple & 0,69 & 0,66 & 0,76 & 0,82 \\
\hline
\end{tabular}

Fuente: Elaboración propia sobre la base de la ENIA, 1979-1999.

\footnotetext{
${ }^{6}$ La tasa de sobrevivencia se calcula como el número de plantas que se mantienen entre $t$ y $t+s$ sobre el número total de plantas existentes en $t$.

${ }^{7}$ Tal comportamiento en estos períodos concuerda con los shocks agregados de la economía chilena. Los períodos de 1979-1984 y 1994-1999 estuvieron caracterizados por crisis económicas; el PIB tuvo una caída de 12,0\% y 0,5\% en 1982 y 1999, respectivamente.

${ }^{8}$ Una relación inversa entre sobrevivencia y tamaño han encontrado, entre otros, Grenaway et al. (2005) para Suecia; Van Biesebroeck (2005) para una muestra de países africanos, y Bernard y Sjöholm (2003) para Irlanda.
} 
cia, pero ésta es común para todos los segmentos de empresas y no sólo para las PYME. En la próxima sección se estudia si estos hallazgos son robustos al controlar por otras características de las plantas y de las industrias.

\section{Metodología}

En este estudio estamos particularmente interesados en analizar qué factores afectan la sobrevivencia de plantas industriales y, especialmente, cómo la influencia del tamaño de las firmas ha cambiado a través del tiempo y sectores. Para esto se estima un modelo Probit para la probabilidad de sobrevivencia, teniendo como variables explicativas características de la planta y del sector industrial donde opera. El modelo a estimar es:

$$
\operatorname{Pr}\left(\mathrm{S}_{\mathrm{ij} \Delta t}\right)=\mathrm{f}\left(\alpha+\beta \mathrm{X}_{\mathrm{it}}+\gamma \mathrm{Z}_{\mathrm{jt}}+\mathrm{d}_{\mathrm{j}}+\mathrm{d}_{\Delta \mathrm{t}}+\varepsilon_{\mathrm{ij} \Delta \mathrm{t}}\right)
$$

donde $\operatorname{Pr}\left(\mathrm{S}_{\mathrm{ij \Delta} \Delta}\right)$ es la probabilidad de sobrevivencia de la planta $i$ localizada en la industria $j$ durante el período $\Delta t ; X_{i t}$ es un vector de variables específicas a la planta y $Z_{j t}$ es un vector de variables sectoriales. $d_{j}$ son los efectos específicos a cada sector, y $d_{\Delta t}$ son efectos específicos para cada período. Todas las variables están medidas en $t$, es decir, al comienzo de cada período. Los sectores industriales están clasificados en 4 dígitos de la Clasificación Internacional Industrial Uniforme (CIIU). Conforme con la evidencia descriptiva presentada, se estima el modelo utilizando períodos de 5 años para calcular la sobrevivencia de las plantas. Para contrastar la robustez de estos resultados, se realizan también estimaciones con períodos de 3 años.

Las variables explicativas a nivel de plantas incluyen una medida de productividad $^{9}$, la intensidad de uso de capital físico y humano, antigüedad y las dos variables categóricas por tamaño. Siguiendo la literatura previa, se espera que las posibilidades de sobrevivir se incrementen con la productividad de la firma. Las intensidades de uso de capital físico y humano se

${ }^{9}$ Para medir la productividad a nivel de la planta se estima una función de producción Cobb-Douglas para cada uno de los sectores manufactureros a tres dígitos de la CIIU. Esto se hace siguiendo la metodología propuesta por Olley y Pakes (1996) y posteriormente modificada por Levinsohn y Petrin (2003a, 2003b), quienes corrigen por el sesgo de simultaneidad, dado que la productividad no es observada por el econometrista pero sí por la firma. Petrin et al. (2004) describen los comandos de Stata para estimar la función de producción y obtener medidas de la productividad total de factores. 
definen como el stock de capital por trabajador y la razón entre los salarios de empleados sobre el total de salarios ${ }^{10}$. Estas variables son incluidas con una aproximación del mix de productos que una planta produce. Existe evidencia de que firmas que producen bienes de mayor calidad —más intensivos en capital físico y humano - tienen una mayor probabilidad de sobrevivir (Bernard y Jensen, 2006).

En el caso de la antigüedad de la planta, se argumenta que, basados en modelos de aprendizaje, la probabilidad de sobrevivencia se incrementa con la edad de la empresa (Jovanovic, 1982). Dado que la ENIA no proporciona información respecto al año de fundación de cada planta, la antigüedad es definida como el número de años desde que la planta aparece en la base de datos.

Potencialmente podría existir una alta correlación entre las variables independientes utilizadas en la estimación. En efecto, plantas más grandes y más antiguas tienden a ser también más productivas. Lo mismo ocurre en el caso de la intensidad de uso de factores. Existe una correlación positiva entre la intensidad de uso de capital —-físico y humano- y la productividad de las plantas. Esto no invalida las conclusiones que surgen del análisis empírico en la medida que el uso de un panel de datos reduce los problemas econométricos asociados a la colinealidad de las variables independientes. Más aún, como se muestra a continuación, los coeficientes de estas variables son estimados con alta precisión.

Las estimaciones incluyen también tres variables de estructura de mercado: la mediana sectorial del tamaño de planta, la intensidad de gasto en publicidad y el índice de concentración de Herfindahl. Siguiendo los modelos de dinámica industrial, estas variables buscan capturar el impacto de las diferencias en los costos de entrada entre sectores. Hopenhayn (1992), por ejemplo, muestra en un modelo de equilibrio general que incrementos en los costos de entrada generan menores tasas de entrada y salida de firmas. Por esto, se espera que la salida de plantas industriales sea menor en sectores con altos costos de entrada. El tamaño de planta sectorial controla por diferencias en la escala mínima eficiente, los gastos en publicidad lo hacen por la importancia de la diferenciación de productos y el índice de concentración, por las diferencias en el grado de competencia doméstica.

${ }^{10} \mathrm{El}$ supuesto es que los empleados tienen un mayor grado de calificación que el promedio de los trabajadores en una planta. Definiciones alternativas de intensidad de uso de capital humano, como por ejemplo la relación entre empleados y obreros, llevan a resultados similares. 


\section{Resultados}

Los resultados de las estimaciones se muestran en el Cuadro $\mathrm{N}^{\circ} 2$ para períodos de 5 y 3 años. El modelo base para ambos grupos de estimaciones, columnas (1) y (4), no incluye las características a nivel de sectores industriales. Las columnas siguientes agregan las variables sectoriales. Finalmente, en las columnas (3) y (6) se incluye una medida de crecimiento de la industria.

En primer lugar, los resultados son concordantes con otros estudios referentes a la sobrevivencia de plantas industriales. Se puede apreciar que las plantas más productivas y antiguas tienen una mayor probabilidad de sobrevivencia. Estos resultados son similares en ambos grupos de estimaciones y para diferentes especificaciones del modelo.

En cuanto a la intensidad de uso de los factores, se encuentra que una mayor intensidad en capital humano no afecta la probabilidad de sobrevivencia, pero en cambio las plantas más intensivas en capital físico exhiben una menor probabilidad de salida ${ }^{11}$. Por su parte, los parámetros estimados para las plantas medianas y pequeñas son negativos y significativos, lo que refleja una mayor salida de plantas de menor tamaño relativo. Según las estimaciones para períodos de 5 años, las plantas medianas y pequeñas tienen, respectivamente, entre $8 \%$ y $16 \%$ de menor probabilidad de sobrevivir que las plantas grandes. De acuerdo a las estimaciones para períodos de 3 años, las plantas medianas y pequeñas tienen entre un 5\% y un $12 \%$ de mayor probabilidad de salida que las plantas grandes.

Una pregunta central es si el efecto del tamaño de las firmas ha cambiado con el tiempo. Como argumentan ciertas líneas de investigación, si las firmas requieren ser grandes para competir exitosamente, entonces el efecto negativo de la variable categórica por tamaño debería aumentar, en valor absoluto, en la medida en que Chile ha ido reduciendo sus barreras al comercio. Para testear la validez de este argumento, se calcula el efecto del tamaño para cada uno de los períodos considerados, aprovechando las ventajas de la no linealidad del modelo de regresión Probit ${ }^{12}$.

${ }^{11}$ Mayor intensidad de capital puede reflejar también mayores costos hundidos, $\mathrm{y}$ por lo tanto menores incentivos a la salida.

${ }^{12}$ En un modelo Probit, el cambio marginal es una función del resto de las variables, y es calculado comúnmente utilizando las medias de todas las otras variables. Para analizar cómo el efecto del tamaño varía entre períodos, calculamos el cambio marginal en la media de las variables, pero cambiando la variable categórica para diferentes períodos. Otra alternativa usada es incluir términos interactivos, sin embargo la interpretación no es simple. El efecto interactivo es también función de otras variables y en la mayoría de los casos es interpretado erróneamente (Ai and Norton, 2003). 


\begin{tabular}{|c|c|c|c|c|c|c|}
\hline \multirow[t]{2}{*}{ Variables } & \multicolumn{3}{|c|}{ Períodos $(\Delta t): 5$ años } & \multicolumn{3}{|c|}{ Períodos $(\Delta t): 3$ años } \\
\hline & (1) & (2) & (3) & (4) & (5) & (6) \\
\hline Productividad & $\begin{array}{c}0,079 \\
(12,71)^{* *}\end{array}$ & $\begin{array}{c}0,079 \\
(12,58)^{* *}\end{array}$ & $\begin{array}{c}0,078 \\
(12,78)^{* *}\end{array}$ & $\begin{array}{c}0,058 \\
(16,00)^{* *}\end{array}$ & $\begin{array}{c}0,058 \\
(15,92)^{* *}\end{array}$ & $\begin{array}{c}0,058 \\
(16,35)^{* *}\end{array}$ \\
\hline KF & $\begin{array}{l}0,019 \\
(3,75)^{* *}\end{array}$ & $\begin{array}{c}0,019 \\
(3,77)^{* *}\end{array}$ & $\begin{array}{c}0,019 \\
(3,77)^{* *}\end{array}$ & $\begin{array}{c}0,014 \\
(5,01)^{* *}\end{array}$ & $\begin{array}{c}0,014 \\
(5,05)^{* *}\end{array}$ & $\begin{array}{c}0,014 \\
(5,01)^{* *}\end{array}$ \\
\hline KH & $\begin{array}{c}-0,007 \\
(0,19)\end{array}$ & $\begin{array}{c}-0,007 \\
(0,19)\end{array}$ & $\begin{array}{c}-0,010 \\
(0,27)\end{array}$ & $\begin{array}{c}-0,008 \\
(0,47)\end{array}$ & $\begin{array}{c}-0,007 \\
(0,40)\end{array}$ & $\begin{array}{l}-0,007 \\
(-0,38)\end{array}$ \\
\hline Antigüedad & $\begin{array}{c}0,056 \\
(10,16)^{* *}\end{array}$ & $\begin{array}{c}0,056 \\
(10,18)^{* *}\end{array}$ & $\begin{array}{c}0,055 \\
(9,79)^{* *}\end{array}$ & $\begin{array}{c}0,036 \\
(9,09)^{* *}\end{array}$ & $\begin{array}{c}0,036 \\
(9,42)^{* *}\end{array}$ & $\begin{array}{c}0,035 \\
(9,17)^{* *}\end{array}$ \\
\hline Pequeñas & $\begin{array}{l}-0,164 \\
(8,21)^{* *}\end{array}$ & $\begin{array}{c}-0,163 \\
(8,22)^{* *}\end{array}$ & $\begin{array}{l}-0,165 \\
(8,20)^{* *}\end{array}$ & $\begin{array}{c}-0,122 \\
(10,36)^{* *}\end{array}$ & $\begin{array}{c}-0,119 \\
(10,39)^{* *}\end{array}$ & $\begin{array}{c}-0,120 \\
(10,44)^{* *}\end{array}$ \\
\hline Medianas & $\begin{array}{l}-0,075 \\
(3,66)^{* *}\end{array}$ & $\begin{array}{l}-0,074 \\
(3,62)^{* *}\end{array}$ & $\begin{array}{l}-0,076 \\
(3,72)^{* *}\end{array}$ & $\begin{array}{l}-0,05 \\
(4,54)^{* *}\end{array}$ & $\begin{array}{l}-0,051 \\
(4,46)^{* *}\end{array}$ & $\begin{array}{c}-0,052 \\
(4,52)^{* *}\end{array}$ \\
\hline Herfindahl & $\begin{array}{l}- \\
-\end{array}$ & $\begin{array}{c}0,424 \\
(2,89)^{* *}\end{array}$ & $\begin{array}{c}0,312 \\
(2,09)^{*}\end{array}$ & $\begin{array}{l}- \\
-\end{array}$ & $\begin{array}{c}0,278 \\
(4,18)^{* *}\end{array}$ & $\begin{array}{c}0,377 \\
(5,03)^{* *}\end{array}$ \\
\hline Mediana empleo sector & $\begin{array}{l}- \\
-\end{array}$ & $\begin{array}{r}0,040 \\
(1,01)\end{array}$ & $\begin{array}{c}0,074 \\
(2,00)^{*}\end{array}$ & - & $\begin{array}{c}0,060 \\
(2,35)^{*}\end{array}$ & $\begin{array}{c}0,062 \\
(2,41)^{*}\end{array}$ \\
\hline Publicidad/ventas & $\begin{array}{l}- \\
-\end{array}$ & $\begin{array}{c}-7,831 \\
(1,90)\end{array}$ & $\begin{array}{c}-5,033 \\
(1,52)\end{array}$ & $\begin{array}{l}- \\
-\end{array}$ & $\begin{array}{c}-0,023 \\
(0,29)\end{array}$ & $\begin{array}{c}-0,026 \\
(0,33)\end{array}$ \\
\hline Crecimiento del sector & - & $\begin{array}{l}- \\
-\end{array}$ & $\begin{array}{c}0,176 \\
(6,78)^{* *}\end{array}$ & $\begin{array}{l}- \\
-\end{array}$ & $\begin{array}{l}- \\
-\end{array}$ & $\begin{array}{c}0,075 \\
(6,16)^{* *}\end{array}$ \\
\hline Observaciones & 16.752 & 6.752 & $0.752 \quad 25$ & 9.052 & .052 & .052 \\
\hline
\end{tabular}

*Significativo al 5\%; ** Significativo al 1\%.

Nota: La variable dependiente es igual a 1 si la planta existe en el año $t$ y en el año $t+5$ o $t+3$ según corresponda, y 0 en cualquier otro caso. Las estimaciones fueron realizadas para cuatro períodos en el caso de intervalos de 5 años: (1979, 1984), (1984, 1989), (1989, 1994), y (1994, 1999), y siete períodos en el caso de intervalos de 3 años: (1979, 1982), (1982, 1985), (1985, 1988), (1988, 1991), (1991, 1994), (1994, 1997) у (1997, 2000). Todas las variables explicativas están medidas en el primer año de cada intervalo. Productividad es el log de la productividad total de los factores. KF es el log del capital por trabajador. $\mathbf{K H}$ es la ratio de los salarios de empleados no dedicados a la producción sobre el total de salarios. Antigüedad es el log de 1+año donde la planta aparece en la base de datos. El índice de Herfindahl mide la concentración sectorial a nivel de 4 dígitos de la CIIU, y se define como la suma del cuadrado de la participación de la producción de cada planta en el total. Pequeñas y medianas son variables categóricas de tamaño (0-50 empleados para pequeñas y 51-150 empleados para medianas). Mediana empleo sector es la mediana del empleo de las plantas pertenecientes a cada sector (4 dígitos CIIU). Publicidad/ventas es la ratio del gasto en publicidad sobre ventas de cada planta, y crecimiento del sector es la variación del empleo sectorial (4 dígitos CIIU) para cada período. Se incluyen también variables categóricas por sector (4 dígitos CIIU) y para cada uno de los períodos en cada regresión. 
Los resultados mostrados en el Cuadro $\mathrm{N}^{0} 3$ sugieren que hay una disminución en la probabilidad de sobrevivencia a través del tiempo. Esto se puede apreciar especialmente en el caso de las plantas pequeñas al comparar los períodos inicial y final: nótese que el hecho de ser pequeña reduce las posibilidades de sobrevivencia en 13\% en el período 1979-1984 (11\% para 1979-1982), y en un 20\% en el período 1994-1999 (17\% en 1997-1999). Los cambios tienden a ser menores en el caso de las plantas medianas: entre ambos períodos extremos de cinco años, la probabilidad de sobrevivencia se redujo de $10 \%$ a $13 \%$.

CUADRO N 3 : $\quad$ CAMBIO EN LA PROBABILIDAD DE SOBREVIVENCIA SEGÚN TAMAÑO Y PERÍODOS

\begin{tabular}{|c|c|c|c|c|c|c|c|}
\hline & \multirow[t]{2}{*}{ Período } & \multicolumn{3}{|c|}{ Pequeñas } & \multicolumn{3}{|c|}{ Medianas } \\
\hline & & $\mathrm{dy} / \mathrm{dx}$ & Interva & о 95\% & $\mathrm{dy} / \mathrm{dx}$ & Intervalc & о $95 \%$ \\
\hline \multirow{4}{*}{ 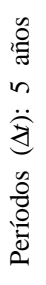 } & 1979-1984 & $\begin{array}{l}-0,13 \\
(8,48)^{* *}\end{array}$ & $-0,16$ & $-0,10$ & $\begin{array}{l}-0,06 \\
(3,46)^{* *}\end{array}$ & $-0,10$ & $-0,02$ \\
\hline & 1984-1989 & $\begin{array}{l}-0,18 \\
(8,54)^{* *}\end{array}$ & $-0,22$ & $-0,14$ & $\begin{array}{l}-0,08 \\
(3,68)^{* *}\end{array}$ & $-0,12$ & $-0,03$ \\
\hline & 1989-1994 & $\begin{array}{c}-0,17 \\
(8,48)\end{array}$ & $-0,21$ & $-0,13$ & $\begin{array}{l}-0,08 \\
(3,66)^{* *}\end{array}$ & $-0,12$ & $-0,03$ \\
\hline & 1994-1999 & $\begin{array}{l}-0,20 \\
(8,30)^{* *}\end{array}$ & $-0,25$ & $-0,15$ & $\begin{array}{l}-0,08 \\
(3,73)^{* *}\end{array}$ & $-0,13$ & $-0,04$ \\
\hline \multirow{7}{*}{ 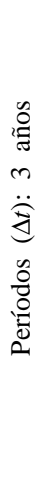 } & 1979-1982 & $\begin{array}{c}-0,11 \\
(10,92)^{* *}\end{array}$ & $-0,13$ & $-0,09$ & $\begin{array}{l}-0,05 \\
(4,40)^{* *}\end{array}$ & $-0,07$ & $-0,03$ \\
\hline & 1982-1985 & $\begin{array}{l}-0,12 \\
(9,70)^{* *}\end{array}$ & $-0,15$ & $-0,10$ & $\begin{array}{l}-0,05 \\
(4,20)^{* *}\end{array}$ & $-0,08$ & $-0,02$ \\
\hline & 1985-1988 & $\begin{array}{c}-0,12 \\
(11,86)^{*}\end{array}$ & $-0,14$ & $-0,10$ & $\begin{array}{l}-0,05 \\
(4,34)^{* *}\end{array}$ & $-0,07$ & $-0,02$ \\
\hline & 1988-1991 & $\begin{array}{l}-0,11 \\
(11,31)^{* *}\end{array}$ & $-0,13$ & $-0,10$ & $\begin{array}{l}-0,05 \\
(4,33)^{* *}\end{array}$ & $-0,07$ & $-0,03$ \\
\hline & 1991-1994 & $\begin{array}{l}-0,12 \\
(9,74)^{* *}\end{array}$ & $-0,14$ & $-0,10$ & $\begin{array}{l}-0,05 \\
(4,22)^{* *}\end{array}$ & $-0,08$ & $-0,03$ \\
\hline & 1994-1997 & $\begin{array}{l}-0,15 \\
(9,89)^{* *}\end{array}$ & $-0,19$ & $-0,13$ & $\begin{array}{l}-, 0,06 \\
(4,37)^{* *}\end{array}$ & $-0,09$ & $-0,04$ \\
\hline & 1997-1999 & $\begin{array}{l}-0,17 \\
(10,31)^{* *}\end{array}$ & $-0,20$ & $-0,14$ & $\begin{array}{l}-0,07 \\
(4,49)^{* *}\end{array}$ & $-0,10$ & $-0,04$ \\
\hline
\end{tabular}

** Significativo al $1 \%$.

Fuente: elaboración propia en base a las estimaciones presentadas en columnas (3) y (6) del Cuadro $\mathrm{N}^{\circ} 2$. 
No obstante estos resultados, no se puede concluir que las probabilidades de sobrevivencia se hayan visto significativamente afectadas para las PYME. Desde un punto de vista estadístico, no se puede rechazar la hipótesis de que estos cambios marginales son iguales para los diferentes períodos bajo estudio. Más aún, si uno compara los períodos más homogéneos en el medio de la muestra, aun cuando existió una profundización de la liberalización comercial, el cambio en la probabilidad de sobrevivencia para las PYME se mantuvo casi inalterado.

A continuación se analiza la relación entre sobrevivencia y liberalización comercial, utilizando diferencias sectoriales en la exposición a la competencia externa. En efecto, se puede argumentar que no todas los sectores son afectados de manera similar por la liberalización, ya que cambios idénticos en los aranceles pueden tener un impacto heterogéneo en los flujos de comercio. Para evaluar el efecto diferenciado entre sectores, se sigue la literatura de grupos de tratamiento y control y se generan dos grupos de sectores industriales según el grado de exposición a la competencia exter$\mathrm{na}^{13}$. Luego se investiga si la sobrevivencia de las diferentes categorías de tamaño de firmas difiere entre sectores más y menos expuestos a la competencia internacional.

Los grupos de tratamiento y control se definen a 4 dígitos de la clasificación CIIU según el promedio de la ratio de comercio internacional (exportaciones más importaciones) en la producción total durante el período bajo estudio. El grupo de tratamiento — sectores más expuestos a la competencia externa - corresponde al tercio de sectores con más alta ratio, mientras que el grupo de control son aquellas industrias en el tercio con la ratio más baja. Luego, se estima el modelo de sobrevivencia incluyendo una variable categórica para las industrias más expuestas a la competencia externa. Para chequear la robustez de estos resultados, se estima también el modelo usando directamente la ratio para todas las industrias.

Debe reconocerse que ésta no es la única manera de distinguir la exposición a la liberalización comercial. De hecho, puede ser perfectamente posible que un sector comercie directamente poco con el resto del mundo, pero que provea de insumos a sectores que sí lo hacen en forma intensiva. Ésta es una hipótesis no fácil de testear empíricamente. El principal problema es que no existen datos de la matriz insumo-producto para sectores a 4 dígitos que potencialmente podrían usarse para ver qué tan expuestos al comercio internacional son los sectores a los cuales una industria provee insumos. Esto implica que nuestro análisis es más relevante para la

${ }^{13}$ Se sigue la metodología presentada por Hanson (2005) para estudiar los efectos de la globalización en el mercado laboral en diferentes regiones de México. 
exposición directa de las plantas al comercio internacional, pero no así para plantas que puedan enfrentar una mayor competencia en sectores relacionados.

Los resultados de las estimaciones, que incluyen una variable de exposición al comercio internacional, se presentan en el Cuadro $\mathrm{N}^{\circ} 4$. En primer lugar, las estimaciones muestran resultados similares según se use una variable categórica o continua para medir las diferencias en el grado de exposición a la competencia internacional de los distintos sectores industriales. En ambos casos, una mayor competencia externa incrementa la probabilidad de sobrevivencia. De acuerdo a la columna (1), las plantas en sectores más expuestos tienen 12,2\% más de probabilidad de sobrevivir que plantas en sectores con baja exposición. Como se observa en las columnas (3) y (4), los resultados son robustos a estimaciones realizadas midiendo la sobrevivencia para períodos de 3 años.

Más interesante es analizar cómo el efecto de ser pequeño y mediano difiere entre sectores industriales. Para esto, calculamos los efectos marginales para ambos grupos de empresas en sectores más y menos expuestos a la competencia externa. Como se muestra en las últimas filas del Cuadro $\mathrm{N}^{\circ} 4$ - columnas (1) y (3) - las empresas pequeñas y medianas tienen menor probabilidad de sobrevivencia, pero no se encuentra evidencia de que esta probabilidad tienda a ser menor en sectores más expuestos a la competencia externa. Más aún, el impacto negativo de ser pequeño tiende a ser menor en las industrias que más comercian con el resto del mundo. En total, el impacto negativo para una planta pequeña sobre la probabilidad de sobrevivencia se reduce en $3 \%$ y $10 \%$ en sectores de alta competencia, pero en $18 \%$ y $12 \%$ en sectores de baja competencia externa. Un resultado similar se da para plantas medianas; la probabilidad de salida se incrementa en $2 \%$ y $4,5 \%$ en sectores de alta competencia, y $10 \%$ y 5,5\% en los de baja competencia.

De este modo, esta evidencia es concordante con la idea de que la globalización no tiene un efecto desproporcionadamente mayor en la sobrevivencia de las empresas de menor tamaño relativo. En comparación con las empresas de igual tamaño en sectores menos expuestos a la competencia externa, plantas pequeñas y medianas tienen mayores probabilidades de sobrevivencia en sectores donde las empresas están más expuestas a la competencia internacional. Esta evidencia tiende a favorecer las hipótesis de Audretsch (2002) respecto a que las PYME pueden jugar un importante rol como fuente de innovación, crecimiento y competitividad en la economía global. 
CUADRO No 4 : SOBREVIVENCIA DE FIRMAS Y COMPETENCIA EXTERNA: MODELO PROBIT

\begin{tabular}{|c|c|c|c|c|}
\hline \multirow[t]{2}{*}{ Variables } & \multicolumn{2}{|c|}{ Períodos $(\Delta t): 5$ años } & \multicolumn{2}{|c|}{ Períodos $(\Delta t)$ : 3 años } \\
\hline & & & & (4) \\
\hline \multirow[t]{2}{*}{ PTF } & 0,081 & 0,080 & 0,059 & 0,061 \\
\hline & $(15,85)^{* *}$ & $(12,56)^{* *}$ & $(16,57)^{* *}$ & $(16,19)^{* *}$ \\
\hline \multirow[t]{2}{*}{ KF } & 0,018 & 0,019 & 0,014 & 0,013 \\
\hline & $(1,77)$ & $(3,66)^{* * *}$ & $(2,54)^{*}$ & $(4,52)^{* *}$ \\
\hline \multirow[t]{2}{*}{ KH } & $-0,063$ & $-0,020$ & $-0,011$ & $-0,008$ \\
\hline & $(0,95)$ & $(0,49)$ & $(0,39)$ & $(0,42)$ \\
\hline Antigüedad & $\begin{array}{c}0,058 \\
(788)^{*}\end{array}$ & 0,058 ** & $\begin{array}{l}0,038 \\
(470) * *\end{array}$ & 0,026 \\
\hline \multirow{2}{*}{ Pequeñas } & $\begin{array}{r}(1,00) \\
-0,167\end{array}$ & $-0,164$ & $-0,113$ & $-0,120$ \\
\hline & $(7,20)^{* *}$ & $(7,96)^{* *}$ & $(8,48)^{* *}$ & $(10,34)^{* *}$ \\
\hline \multirow[t]{2}{*}{ Medianas } & $-0,090$ & $-0,076$ & $-0,052$ & $-0,053$ \\
\hline & $(2,78)^{* *}$ & $(3,53)^{* *}$ & $(2,90)^{*}$ & $(4,36)^{* *}$ \\
\hline \multirow[t]{2}{*}{ Índice Herfindahl } & $-0,077$ & 0,353 & 0,346 & 0,448 \\
\hline & $(0,48)$ & $(2,30)^{*}$ & $(3,81)^{* *}$ & $(5,36)^{* *}$ \\
\hline \multirow[t]{2}{*}{ Mediana empleo sector } & 0,059 & 0,075 & 0,059 & 0,072 \\
\hline & $(1,05)$ & $(1,93)$ & $(1,75)$ & $(5,36)^{* *}$ \\
\hline \multirow[t]{2}{*}{ Publicidad / ventas } & $-3,686$ & $-5,607$ & 0,049 & $-0,018$ \\
\hline & $(0,92)$ & $(1,69)$ & $(0,55)$ & $(0,22)$ \\
\hline \multirow[t]{2}{*}{ Crecimiento del sector } & 0,235 & 0,185 & 0,063 & 0,074 \\
\hline & $(9,71)^{* *}$ & $(6,67)^{* *}$ & $(4,45)^{* *}$ & $(5,36)^{* *}$ \\
\hline \multirow[t]{2}{*}{ Alta competencia externa } & 0,122 & - & 0,039 & \\
\hline & $(3,21)^{* *}$ & - & $(1,95)^{*}$ & \\
\hline Competencia externa & & $\begin{array}{l}0,035 \\
(7,36)^{* *}\end{array}$ & & $\begin{array}{c}0,025 \\
(10,85)\end{array}$ \\
\hline Observaciones & 7361 & 15976 & 13004 & 6858 \\
\hline \multicolumn{5}{|l|}{ Efecto marginal: Pequeñas } \\
\hline \multirow[t]{2}{*}{ Alta competencia } & $-0,03$ & - & $-0,100$ & \\
\hline & $(3,23)^{* *}$ & - & $(9,27)^{* *}$ & \\
\hline \multirow[t]{2}{*}{ Baja competencia } & $-0,18$ & - & $-0,121$ & \\
\hline & $(8,26)^{* *}$ & - & $(9,31)^{* *}$ & \\
\hline \multicolumn{5}{|l|}{ Efecto marginal: Medianas } \\
\hline \multirow[t]{2}{*}{ Alta competencia } & $-0,02$ & - & $-0,045$ & \\
\hline & $(1,78)$ & - & $(2,67)^{* *}$ & \\
\hline \multirow[t]{2}{*}{ Baja competencia } & $-0,10$ & - & $-0,055$ & \\
\hline & $(2,69)^{* *}$ & - & $(2,75)^{* *}$ & \\
\hline
\end{tabular}

* Significativo al 5\%; ** Significativo al 1\%.

Nota: La variable dependiente es igual a 1 si la planta existe en el año $t$ y en el año $t+5$ o $t+3$ según corresponda, y 0 en cualquier otro caso. Las estimaciones fueron realizadas para cuatro períodos en el caso de intervalos de 5 años: (1979, 1984), (1984, 1989), (1989, 1994) y (1994, 1999), y siete períodos en el caso de intervalos de 3 años: (1979, 1982), (1982, 1985), (1985, 1988), (1988, 1991), (1991, 1994), (1994, 1997) у (1997, 2000). Todas las variables explicativas están medidas en el primer año de cada intervalo. Productividad es el log de la productividad total de los factores. KF es el log del capital por trabajador. KH es la ratio de los salarios de empleados no dedicados a la producción sobre el total de salarios. Antigüedad es el log de 1+año donde la planta aparece en la base de datos. El índice de Herfindahl mide la concentración sectorial a nivel de 4 dígitos de la CIIU, y se define como la suma del cuadrado de la participación de la producción de cada planta en el total. Pequeñas y medianas son variables categóricas según tamaño (0-50 empleados para pequeñas y 51-150 empleados para medianas). Mediana empleo sector es la mediana del empleo de las plantas pertenecientes a cada sector (4 dígitos CIIU). Publicidad/ventas es la ratio del gasto en publicidad sobre ventas de cada planta, y crecimiento del sector es la variación del empleo sectorial (4 dígitos CIIU) para cada período. La variable competencia externa corresponde al promedio de la ratio de exportaciones más importaciones sobre producción. Alta competencia es una variable categórica igual a 1 para plantas pertenecientes al tercio superior de la variable competencia externa, y 0 si está en el tercio inferior. 
Un ejercicio final que realizamos fue estimar el modelo para diferentes sectores. Esto es útil para entregar información de cómo la probabilidad de sobrevivencia de las PYME difiere por industrias manufactureras. En general, cuando se postula que los gobiernos deben destinar recursos al fomento de estas empresas no se analizan las diferencias existentes entre distintos sectores.

En el Cuadro $\mathrm{N}^{\circ} 5$ se presentan las estimaciones para 25 sectores manufactureros a 3 dígitos CIIU ${ }^{14}$. Para la mayoría de los sectores (16 sobre 25) se encuentra una relación negativa y significativa entre nuestra variable categórica para plantas pequeñas y su probabilidad de sobrevivencia. En cambio, en sólo 8 de los 25 sectores esta relación es negativa para plantas medianas. Existe también una alta heterogeneidad a través de los sectores. Por ejemplo, en el caso del sector alimentos, las plantas pequeñas tienen un $14 \%$ menos de probabilidad de sobrevivir que las plantas grandes. En el caso de las plantas medianas, es un $8 \%$ menos. En el caso del sector químico, la desventaja de ser pequeño tiende a ser más pronunciada. Las plantas pequeñas y medianas tienen una probabilidad 30\% y $20 \%$ menor de sobrevivencia que las grandes empresas, respectivamente. Sin embargo, en varios sectores no se puede rechazar la hipótesis de que la probabilidad de sobrevivencia no difiere por tamaño de plantas. Éste es el caso de algunos sectores, como bebidas y calzado, en los cuales las variables categóricas para pequeñas y medianas empresas son no significativas.

En resumen, la evidencia sectorial sugiere que la relación negativa entre sobrevivencia y tamaño no es necesariamente válida para todos los sectores industriales. En base a ello, este trabajo sugiere algunas dudas respecto a la implementación de políticas de apoyo a las PYME que no consideran las diferentes realidades sectoriales ni las diferencias entre plantas pequeñas y medianas.

¿Qué puede explicar estas diferencias entre sectores? Existen varias hipótesis alternativas susceptibles de ser analizadas en mayor profundidad, pero que van más allá del objetivo de este trabajo. Una explicación simple tiene que ver con el potencial aprovechamiento de economías de escala. En aquellos sectores donde la escala mínima eficiente es relativamente grande,

${ }^{14}$ La clasificación contempla 28 sectores manufactureros, pero para 3 de ellos el reducido número de observaciones no permite realizar estas estimaciones. Por consideraciones de espacio, y dado que las estimaciones usando períodos de tres años son similares, sólo presentamos los resultados para períodos de cinco años. Por la misma razón sólo presentamos el coeficiente de las variables categóricas por tamaño. El modelo, sin embargo, ha sido estimado controlando por las otras características de las plantas, de las industrias y por variables categóricas por período. 


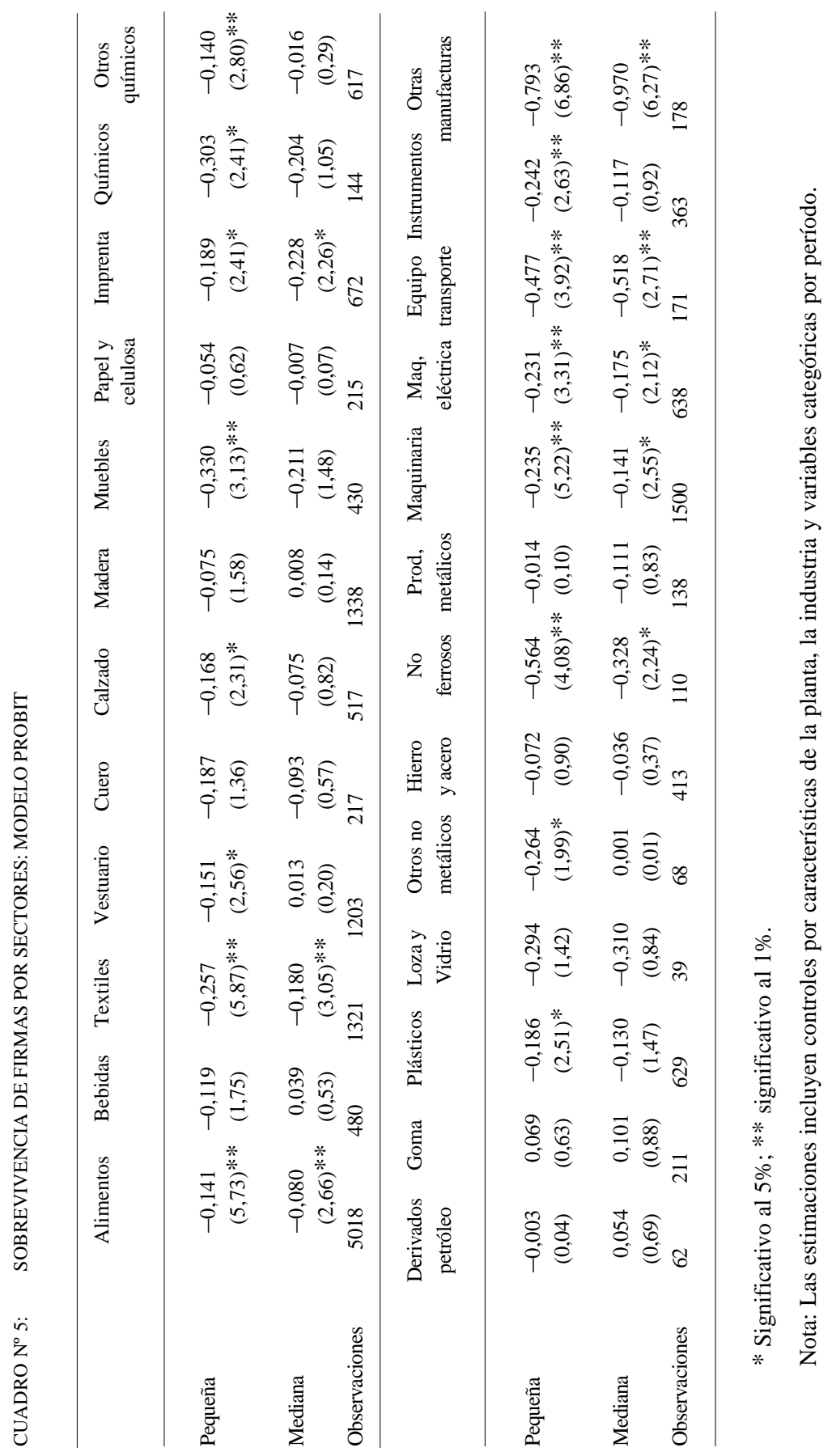


las PYME estarían en desventaja respecto a firmas más grandes. En efecto, Alvarez y Crespi (2001) encuentran que las PYME tienen una menor presencia en sectores donde las economías de escala tienden a ser más importantes. Otro argumento ha sido proporcionado por Audretsch (2002) y se refiere al hecho de que las firmas pequeñas podrían tener ventajas en aquellos sectores altamente innovadores, en los cuales las ventajas de escala son de menor importancia. Incluso, siguiendo a Rajan y Zingales (1998), se podría argumentar que si son los problemas de acceso al crédito los que afectan mayormente a las empresas pequeñas, entonces las PYME podrían ser potencialmente más desfavorecidas en aquellos sectores manufactureros donde las necesidades de financiamiento son mayormente cubiertas por fondos externos a la firma. En resumen, varias razones podrían explicar las diferencias sectoriales encontradas en la relación de probabilidad de muerte y tamaño de las plantas, lo que abre interesantes preguntas para una futura investigación de estos temas.

\section{Conclusiones}

Hay diferentes visiones respecto a cómo la sobrevivencia de las PYME se ve afectada por la creciente liberalización de las economías, lo que genera a su vez diferentes presiones sobre las políticas gubernamentales para aumentar su competitividad. No obstante, la evidencia empírica es limitada para los países en desarrollo. Este estudio entrega evidencia respecto de la sobrevivencia de las PYME en un período largo, en orden a mostrar cómo su desempeño ha cambiado a través del tiempo y entre sectores.

El principal objetivo de este estudio es analizar si el proceso de liberalización ha tenido un efecto desproporcionadamente negativo sobre las PYME. En efecto, la creencia de que para competir internacionalmente es necesario ser grande implicaría que las empresas más pequeñas serían más afectadas por la liberalización. Los resultados de este trabajo no confirman esta visión. Por el contrario, la evidencia presentada muestra que las empresas más pequeñas no han sido dramáticamente afectadas por la mayor exposición a la competencia externa.

El análisis entre sectores — dependiendo de su grado de exposición a la competencia internacional- muestra que las PYME no han sido altamente afectadas por la liberalización de la economía, contraviniendo algunas creencias populares en torno a este fenómeno. Se encuentra que, en comparación con las empresas de igual tamaño en sectores menos expuestos a la competencia externa, plantas pequeñas y medianas tienen mayores 
probabilidades de sobrevivencia en sectores donde las empresas están más expuestas a la competencia internacional. Basado en lo anterior, la conclusión general del estudio es que las PYME pueden, efectivamente, aprovechar sus ventajas comparativas y tener un rol importante en la economía global.

Nuestros resultados para los 25 sectores manufactureros muestran una alta heterogeneidad en el impacto que tiene el tamaño en la sobrevivencia. Esta evidencia sugiere algunas dudas respecto a la implementación de políticas de apoyo a las PYME que no consideran las diferentes realidades sectoriales, ni las diferencias entre plantas pequeñas y medianas.

\section{REFERENCIAS}

Acs, Z., R. Morck, M. Shaver y B. Yeung (1996): “The Internationalization of Small and Medium-Sized Enterprises: A Policy Perspective”. En Small Business Economics, 9 (1): 7-20.

Ai, Ch. y E. C. Norton (2003): "Interaction Terms in Logit and Probit Models". En Economics Letters, 80: 123-129.

Alarcón, C. y G. Stumpo (2001): “Políticas para Pequeñas y Medianas Empresas en Chile”. En Revista de la CEPAL, No 74, agosto.

Álvarez, R. y G. Crespi (2001): "La Importancia Relativa de las Pequeñas y Medianas Empresas: Un Análisis de sus Determinantes en la Industria Manufacturera”. Cuadernos de Economía, 38: 347-365.

Álvarez, R. y H. Görg (2007): "Multinationals as Stabilizers? Economic Crisis and Plant Employment Growth”. Documento de Trabajo No 412, Banco Central de Chile.

Álvarez, R. y S. Vergara (2006): "Globalization, Survival, and Growth of Small and Medium-Sized Firms”. Febrero, disponible en http://ssrn.com/abstract=880785.

Audretsch, D. (2002): “The Dynamic Role of Small Firms: Evidence from the U.S.”. En Small Business Economics, Vol. 18, 13-40. (1995): Innovation and Industry Evolution. Cambridge, MA: MIT Press.

Benavente, J. M., A. Galetovic y R. Sanhueza (2005): "La Dinámica Industrial y la Financiación de las Pyme”. En El Trimestre Económico, LXXII(286): 217-254.

Benavente, J. M. y Ferrada (2003): "Probability of Survival of New Manufacturing Plants: The Case of Chile”. Mimeo, Universidad de Chile.

Bernard, A. B. y J. B. Jensen (2006): "Firm Structure, Multinationals and Manufacturing Plants Deaths". En Review of Economics and Statistics, por aparecer.

Bernard, A. B. y F. Sjöholm (2003): "Foreign Owners and Plant Survival”. NBER Working Paper $\mathrm{N}^{\circ} 10039$, October.

Cabrera, A., S. de la Cuadra, A. Galetovic y R. Sanhueza (2002): "Las PYME: ¿Quiénes Son, Cómo Son, y Qué Hacer con Ellas?”. Sofofa, Chile.

Chandler, A. (1990). Scale and Scope: The Dynamics of Industrial Capitalism. Cambridge, MA: Harvard University Press.

Cohen, W. y S. Kepler (1996): “A Reprise of Size and R\&D”. En Economic Journal, 106, 925-951. 
Cooley, T. y V. Quadrini (2001): “Financial Markets and Firm Dynamics”. En American Economic Review, 91 (5): 1286-1310.

Greenaway, D., J. Gullstrand y R. Kneller (2005): “Surviving Globalisation”. GEP Research Paper 05, University of Nottingham.

Hanson, G. (2005): “Globalization, Labor Income, and Poverty in Mexico”. Working Paper NBER, No 11027, enero.

Hopenhayn, H. (1992): “Entry, Exit, and Firm Dynamics in Long Run Equilibrium”. En Econometrica, 60 (2): 1127-1150.

Jovanovic, B. (1982): "Selection and Evolution of Industries”. En Econometrica, 50 (3): 649-670.

Levinsohn, J. y A. Petrin (2003a): "Estimating Production Functions Using Inputs to Control for Unobservables”. En Review of Economic Studies, 70 (3): 317-341. (2003b): “On the Micro-Foundations of Productivity Growth”. Mimeo, University of Chicago.

Olley, S. y A. Pakes (1996): "The Dynamics of Productivity in the Telecommunications Equipment Industry”. En Econometrica, 64 (5): 1263-1297.

Peres, W. y Stumpo, G. (2000): "Small and Medium-Size Manufacturing Enterprises in Latin America and the Caribbean under the New Economic Model”. En World Development, 28 (9), 1643-1655.

Petrin, A., B. P. Poi y J. Levinsohn (2004): "Production Function Estimation in Stata Using Inputs to Control for Unobservables”. En Stata Journal 4 (2): 113-12

Rajan, R, y L. Zingales (1998): "Financial Dependence and Growth". En American Economic Review 88 (3): 559-586.

UNCTAD (2004): "Promoting the Export Competitiveness of SMEs". Mimeo, United Nations Conference on Trade and Development, octubre.

Van Biesebroeck, J. (2005): "Firm Size Matters: Growth and Productivity Growth in African Manufacturing”. En Economic Development and Cultural Change, 53, 545-583. 